\title{
The Changes of Cancer Patients' Perception, Attitude and Clinical Practice in Complementary and Alternative Medicine during COVID-19: A multicenter before-after cross-sectional study
}

\section{Yichao Wang ( $\square$ wangyc128@aliyun.com )}

Yueyang Hospital of Integrated Traditional Chinese and Western Medicine, Shanghai University of

Traditional Chinese Medicine

\section{Geliang Yang}

Shanghai Clinical Research Center

\section{Zhan Gu}

Shanghai Pulmonary Hospital, Tongji University

\section{Chengyan Wang}

Yueyang Hospital of Integrated Traditional Chinese and Western Medicine, Shanghai University of

Traditional Chinese Medicine

\section{Huiqing Zhang}

Changhai Hospital, Naval Medical University

\section{Ling Xu}

Yueyang Hospital of Integrated Traditional Chinese and Western Medicine, Shanghai University of Traditional Chinese Medicine

\section{Research Article}

Keywords: Complementary and alternative medicine, Cancer, COVID-19, survey, medical pattern

Posted Date: September 30th, 2021

DOl: https://doi.org/10.21203/rs.3.rs-832424/v2

License: (c) (1) This work is licensed under a Creative Commons Attribution 4.0 International License. Read Full License 


\section{Abstract \\ Background}

With the rapid spread of COVID-19 worldwide, the clinical practice in cancer treatment of complementary and alternative medicine (CAM) becomes significantly different. This study was designed to explore the changes of China's cancer patients' perception, attitude and clinical practice in CAM after the pandemic of COVID-19.

\section{Methods}

A multicenter before-after cross-sectional study was conducted, including 448 cancer patients by a survey.

\section{Results}

For perception, half of the cancer patients (51.2\%) reported they had received adequate information in CAM cancer treatment and had a great expectation in medical appointment pattern (40.2\%). For attitudes, most of the cancer patients $(79.4 \%)$ had full of confidence in CAM cancer treatment and showed a strong desire to learn more about CAM (70.2\%). Nearly half of the cancer patients (44.0\%) presented their oncology clinics had been greatly affected by COVID-19, however, most (82.8\%) refused to the online medical service pattern. For clinical practice, Chinese herbal medicine was widely used before (89.5\%) / after $(90.3 \%)$ the epidemic of COVID-19 and the main purpose of receiving CAM therapy was to improve immune system (71.8\%). The frequency of patients' visiting the clinic decreased significantly $(P<0.001)$, and most $(90.0 \%)$ didn't change their follow-up pattern. The majority of the cancer patients (83.3\%) were identified as CAM users and the only predictive factor was national health insurance in the multivariable logistic regression model.

\section{Conclusion}

China's cancer patients show more interest and confidence in treating cancer by CAM, and the new medical pattern such as medical appointment, has been gradually accepted during the COVID-19 pandemic. However, the cancer patients' perception in CAM and online medical service pattern still need to be improved.

\section{Background}

According to the Report on Global Cancer Statistics 2020, a total of 19.3 million new cancer cases and almost 10.0 million deaths occurred worldwide in 185 countries [1]. The usage of complementary and 
alternative medicine (CAM) in cancer treatment has grown rapidly in the past decade, $33.3 \%$ of cancer patients had received CAM therapy in the United States while $40.0-83.0 \%$ in China [2-5].

Since the rapid spread of novel coronavirus disease (COVID-19) in 2020 worldwide, it has been a huge challenge to the clinical practice of cancer patients and oncologists, especially for CAM [6], which contains lots of close-contact therapies such as acupuncture and massage. During the epidemic of COVID-19, cancer itself along with its treatment makes cancer patients more susceptible to pneumonias, due to weakened immune response to respiratory bacteria and virus, meanwhile, the COVID-19 forces most hospitals in the epidemic areas to reduce non-emergency admissions and services, which led to the difficulties in visiting inpatients, outpatients and follow-up [7]. In addition, CAM, mainly included traditional Chinese medicine (TCM) and Chinese patented medicine, was directly applied against COVID19 in China [8-11].

The COVID-19 might significantly change the clinical practice in cancer treatment of CAM. It was estimated that the drop-out rate of clinical trials during the COVID-19 pandemic was about $70-80 \%$, and Yang, et al predicted that the next five years might witness a sharp fall in the number of the clinical trials of integrative cancer therapies [12]. Whether and how the cancer patients' view and use CAM during the epidemic of COVID-19 in China remain unclear. The purpose of this study was to explore the changes of China's cancer patients' perception, attitude and clinical practice in CAM after the epidemic of COVID-19.

\section{Methods}

\section{Participants}

The multicenter cross-sectional study was conducted at five large-scale centers in Shanghai, China from July 2020 to December 2020. The qualified outpatients who were 18 years and older, speaking Chinese and with clear cancer diagnosis signed an informed consent and were required to finish the survey. The inclusion and exclusion of the cancer patients were analyzed and showed as the flowcharts. The study was approved by Ethics Committee of Yueyang Hospital, Shanghai University of Traditional Chinese Medicine (2020-073).

\section{Surveys}

The online survey was designed through a comprehensive review of the literature and discussions with experienced medical oncologists and CAM physicians. The survey was repeatedly modified and divided into three sections according to feedback of the consultants. In the first section, the participants were required to provide demographic information: age, gender, education background, health insurance, annual household income, type of cancer, Eastern Cooperation Oncology Group - performance status (ECOG-PS) score, time of first diagnosis and clinical stage. Then the patients would be asked whether they had ever used CAM to treat cancer? If patients chose "No", the survey would be terminated, and the patients would be required to give reasons, including (1) do not believe CAM, (2) do not know CAM, (3) 
not interested in CAM, (4) too expensive, (5) CAM is ineffective in treating cancer, and (6) other reasons. CAM is defined as a group of diverse medical and health care interventions, practices, products, or disciplines according to the National Center for Complementary and Integrative Health (NCCIH), and it is mainly consisted of Chinese herbal medicine/Chinese patent medicine, diet therapy, acupuncture, Massage, Taichi, Qigong in this survey [3]. If patients chose "Yes", they would enter the second section, focused on the perceptions and clinical practice of the cancer patients toward CAM before and after the epidemic of COVID-19. Questions in this section mainly concerned on changes in cancer treatments (conventional medicine and CAM therapies, aim of using CAM, CAM oncologist/therapist, frequency of visits and cost of CAM therapy) and medical pattern of CAM (online medical service, medical appointment, home visit and hospital visit). The final section dealt with the attitudes of the cancer patients towards CAM treatments and medical pattern by using a Likert scale. The Likert scale was dichotomized to be able to compare the responses; strongly agree and agree were grouped as yes while a neutral response, disagree, and strongly disagree were grouped as a no.

\section{Statistical Analysis}

Descriptive statistics (frequency distribution, mean \pm standard deviation [SD]) were used to summarize patients' baseline characteristics. Differences between the cancer patients (CAM users vs non-CAM users and before COVID-19 vs after the epidemic of COVID-19) were assessed by Student's $t$ test, chi-square test, and Mann-Whitney $\mathrm{U}$ test. Univariate and multivariable analysis were used to determine the associations of baseline characteristics and other relevant factors. Multivariate logistic regression model began with all variables having a $P$-values $<0.25$ from the univariate analysis. Age and sex were kept in the model regardless of their significance. All the difference was considered to be significant when $P<$ 0.05. All analyses were processed by SPSS version 25.0 (SPSS Inc., Chicago, IL, USA).

\section{Results}

\section{Baseline Demographic Information}

A total of 510 questionnaires were sent to the cancer patients, and 448 questionnaires were valid for final analysis, others were excluded due to the incompleteness filling. The majority of cancer patients $(373 / 448,83.3 \%)$ had used CAM at least one time, and the others $(75 / 448,16.7 \%)$ were non-CAM users.

All of the demographic and diagnosis information is presented in Table 1. For the CAM users, the average age was 63.7 years (SD, 9.5), with more woman (59.8\%) participated. Most of the patients $(68.4 \%)$ completed high school, and $20.6 \%$ graduated from college or university. The mean disease duration time was 41.4 months (SD, 25.8). More than half of the CAM users were at clinical stage I $(55.5 \%)$ and ECOGPS score 1 (59.8\%). Lung cancer (74.5\%) was the most common cancer, followed by colorectal $(10.5 \%)$ and stomach (5.6\%).

Table 1 Characteristics of Cancer Patients $(\mathrm{N}=448)$. 


\begin{tabular}{|c|c|c|c|}
\hline Variable & $\begin{array}{l}\text { CAM Users } \\
(n=373)\end{array}$ & $\begin{array}{l}\text { Non-CAM Users } \\
(n=75)\end{array}$ & $P$-value \\
\hline Age, years, mean $\pm S D$ & $63.7 \pm 9.5$ & $58.9 \pm 11.4$ & 0.006 \\
\hline Sex, n (\%) & & & 0.857 \\
\hline Male & $150(40.2)$ & $31(41.3)$ & \\
\hline Female & $223(59.8)$ & $44(58.7)$ & \\
\hline Education, n (\%) & & & 0.003 \\
\hline Primary school & $41(11.0)$ & $13(17.3)$ & \\
\hline High school & $255(68.4)$ & $57(76.0)$ & \\
\hline College or university & $77(20.6)$ & $5(6.7)$ & \\
\hline Annual household income, \$, n (\%) & & & 0.109 \\
\hline$<12000$ & $60(16.1)$ & $17(22.7)$ & \\
\hline $12000-18000$ & $202(54.2)$ & $40(53.3)$ & \\
\hline $18000-30000$ & $81(21.7)$ & $16(21.3)$ & \\
\hline $30000-45000$ & $21(5.6)$ & $2(2.7)$ & \\
\hline $45000-15000$ & $6(1.6)$ & $0(0.0)$ & \\
\hline$>150000$ & $3(0.8)$ & $0(0.0)$ & \\
\hline Health insurance, n (\%) & & & $<0.001$ \\
\hline National medical card & $305(81.8)$ & $39(52.0)$ & \\
\hline Non-national medical card & $68(18.2)$ & $36(48.0)$ & \\
\hline Disease duration, months, mean $\pm S D$ & $41.4 \pm 25.8$ & $34.2 \pm 15.6$ & 0.015 \\
\hline Cancer stage, n (\%) & & & 0.455 \\
\hline Stage I & $207(55.5)$ & $33(44.0)$ & \\
\hline Stage II & $56(15.0)$ & $22(29.3)$ & \\
\hline Stage III & $51(13.7)$ & 14 (18.7) & \\
\hline Stage IV & $59(15.8)$ & $6(8.0)$ & \\
\hline
\end{tabular}

Table 1 (continued). 


\begin{tabular}{|llll|}
\hline Variable & $\begin{array}{l}\text { CAM Users } \\
(\mathbf{n}=373)\end{array}$ & $\begin{array}{l}\text { Non-CAM Users } \\
(\mathbf{n}=75)\end{array}$ & P-value \\
\hline ECOG - PS score & & & 0.072 \\
\hline 0 & $118(31.6)$ & $33(44.0)$ & \\
\hline 1 & $223(59.8)$ & $36(48.0)$ & \\
\hline 2 & $30(8.0)$ & $4(5.3)$ & 0.469 \\
\hline 3 & $2(0.5)$ & $2(2.7)$ & \\
\hline Cancer type, $\mathbf{n}(\%)$ & & & \\
\hline Lung & $278(74.5)$ & $60(16.1)$ & \\
\hline Colorectal & $39(10.5)$ & $5(1.3)$ & \\
\hline Stomach & $21(5.6)$ & $1(0.3)$ & \\
\hline Liver & $14(3.8)$ & $4(1.1)$ & \\
\hline Breast & $11(2.9)$ & $3(0.8)$ & $1(0.3)$ \\
\hline Ovary & $9(2.4)$ & $1(0.3)$ & $0(0)$ \\
\hline Cervix & $5(1.3)$ & $1(0.3)$ & $0(0.0)$ \\
\hline Kidney & $5(1.3)$ & $0(0.0)$ & \\
\hline Esophagus & $3(0.8)$ & $3(0.0)$ & \\
\hline Pancreas & $3(0.8)$ & $3(0.8)$ & \\
\hline Bladder & $2(0.5)$ & $(1.6)$ & \\
\hline Prostate & & \\
\hline Other & & \\
\hline
\end{tabular}

Abbreviations: CAM: complementary and alternative medicine; ECOG: Eastern Cooperative Oncology Group; PS: performance status; SD: Standard deviation.

Among the non-CAM users, four did not believe in CAM, 39 did not know enough about CAM, 14 had no interest, seven were concerned about the cost, seven reported CAM was declined by their oncologists and 16 felt very difficult to take CAM therapies. More than half of the non-CAM users (68.0\%) reported would like to try CAM therapy in the future.

By multivariable logistic regression analyses, CAM users were predicted only by national health insurance (odds ratio $[\mathrm{OR}]=3.47,95 \% \mathrm{Cl}=2.00-5.99$ ), which is provided in Table 2 . 
Table 2 Multivariable Analysis of Factors Predictive of Patients' CAM Use ( $N=448)$.

\begin{tabular}{|c|c|c|c|}
\hline \multirow[t]{2}{*}{ Predictor } & \multicolumn{3}{|c|}{ Received CAM Treatment } \\
\hline & $\begin{array}{l}\text { Odds } \\
\text { Ratio }\end{array}$ & $95 \% \mathrm{Cl}$ & $P$-value \\
\hline Age ( $\geq 65$ vs $<65$ years $)$ & 1.61 & $\begin{array}{l}0.94- \\
2.76\end{array}$ & 0.082 \\
\hline Sex (female vs male) & 1.22 & $\begin{array}{l}0.71- \\
2.10\end{array}$ & 0.469 \\
\hline Education (primary and high school vs college and university) & 2.64 & $\begin{array}{l}0.96- \\
7.30\end{array}$ & 0.061 \\
\hline Annual household income ( $\geq 30000$ vs $<30000)$ & 1.65 & $\begin{array}{l}0.34- \\
8.11\end{array}$ & 0.539 \\
\hline $\begin{array}{l}\text { Health insurance (national medical card vs non-national medical } \\
\text { card) }\end{array}$ & 3.47 & $\begin{array}{l}2.00- \\
5.99\end{array}$ & $<0.001$ \\
\hline Disease duration ( $\geq 40$ vs $<40$ months) & 1.38 & $\begin{array}{l}0.79- \\
2.43\end{array}$ & 0.264 \\
\hline ECOG - PS score (0 - 1 vs 2 - 3 ) & 1.28 & $\begin{array}{l}0.49- \\
3.38\end{array}$ & 0.612 \\
\hline
\end{tabular}

Abbreviations: CAM: complementary and alternative medicine; Cl: confidence interval; ECOG: Eastern Cooperative Oncology Group; PS: performance status.

\section{Perception}

Cancer patients' perception in CAM treatments and medical pattern is presented in Table 3. Half of the cancer patients $(51.2 \%)$ presented that they had received adequate information in CAM cancer treatments. Sixty-eight patients (18.2\%) thought CAM would cause negative effects and 33 patients (8.8\%) had once experienced any side effects. Among those patients, the most frequent side effect events were stomachache (30.3\%) and abnormal liver function (30.3\%), followed by lack of appetite $(12.1 \%)$ and diarrhea (12.1\%). For medical pattern, most of patients believed medical appointment $(40.2 \%)$ and online medical service (27.6\%) might be widely implemented in the future. However, most of the patients could not predict when CAM oncology clinic recover.

Table 3 Perceptions of the cancer patients who used CAM $(\mathrm{N}=373)$. 
1. Have you received adequate information in CAM cancer treatment?

1. Do you think CAM therapies would cause negative effects?

1. Do you experience any negative effects from CAM?

1. Which of the following negative effect/effects caused by CAM have you ever experienced?

Abnormal liver function

Stomachache

Lack of appetite

Diarrhea

Myelosuppression

Fatigue

Table 3 (continued). 
1. Which of the following negative effect/effects caused by CAM have you ever experienced? (continued).

Nausea/vomiting

Constipation

Cardiac arrhythmias

Other

1. After the epidemic of COVID-19, do you think there will be any changes in the pattern of CAM oncology clinic in future?

Medical appointment

Online medical service

Home visit

No change

I don't know

Other

1. Once the COVID-19 is under control, how long do you predict the CAM oncology clinic will recover to normal?

Regard the current pattern as normal during the COVID-19 pandemic

1-3 months

4-6 months

7-8 months

9-12 months

More than 12 months 
Abbreviations: CAM: complementary and alternative medicine; COVID-19: coronavirus disease 2019.

\section{Attitudes}

A Likert scale is used to present the attitudes of cancer patients towards CAM and medical pattern after the epidemic of COVID-19 (Fig. 1). After witnessed the better efficacy of CAM in treating COVID-19 [13], 296 cancer patients $(79.4 \%)$ had full of confidence in CAM which would be also effective in treating cancer and 262 cancer patients $(70.2 \%)$ showed a strong desire to learn more about CAM in the future. For medical pattern, nearly half of the cancer patients (44.0\%) thought their oncology clinics had been greatly affected due to the COVID-19. However, when they faced to the online medical service pattern, strongly promoted by the government and hospitals to provide convenient medical services, most of the cancer patients $(82.8 \%)$ refused. Nevertheless, the same as online service, online appointment seemed to receive more favor $(55.0 \%)$ and might have broad application prospect in the future.

\section{Clinical practice}

All the questions listed in Table 4 are about the clinical practice of the cancer patients in CAM. Chinese herbal medicine was widely used in cancer patients before (89.5\%) / after $(90.3 \%)$ the epidemic of COVID19 , followed by proprietary Chinese medicine before (20.4\%) / after (21.2\%) the epidemic of COVID-19. The utilization rate of acupuncture and moxibustion therapy was slightly declined from $3.2 \%$ to $2.4 \%$. The main purpose of receiving CAM therapy was to improve immune system $(71.8 \%)$ and prevent recurrence and metastasis after surgery (61.1\%). Comparing to the time before the epidemic of COVID-19, the frequency of patients' visiting the clinic decreased significantly. Despite the challenges of COVID-19, most patients didn't change their CAM oncologists/therapists (74.0\%) or follow-up pattern (90.0\%).

Table 4 Clinical practice of the cancer patients who used CAM $(N=373)$. 
Questions

\section{Which kinds of CAM cancer treatment did you use?}

Chinese herbal medicine

Proprietary Chinese medicine

Acupuncture and moxibustion therapy

Diet-based therapy

Chi gong

Massage therapy

Tai chi

Other
Before COVID-

$19, \mathrm{~N}(\%)$
Since COVID-19,

$\mathrm{N}(\%)$
$P$ value
$334(89.5)$

76 (20.4)

$12(3.2)$

$8(2.1)$

$6(1.6)$

$0(0.0)$

$3(0.8)$

$1(0.3)$
$337(90.3)$

79 (21.2)

$9(2.4)$

$8(2.1)$

$9(2.4)$

$0(0.0)$

$4(1.1)$

$1(0.3)$

\section{What was your purpose of using CAM?}

\begin{tabular}{lll} 
Prevent recurrence and metastasis after surgery & $231(61.9)$ & $228(61.1)$ \\
\hline Improve immune system & $260(69.7)$ & $268(71.8)$ \\
\hline Manage symptoms & $100(26.8)$ & $108(29.0)$ \\
\hline Increase the effect of conventional treatment & $52(13.9)$ & $58(15.5)$ \\
\hline Improve quality of life & $42(11.3)$ & $46(12.3)$ \\
\hline Not clear & $6(1.6)$ & $2(0.5)$
\end{tabular}

\section{How often did you come to the CAM}

oncology clinic?

Once a week

Twice a week

Thrice a week
$2(0.5)$

$250(67.0)$

$18(4.8)$

\section{Table 4 (continued).}


Questions

3. How often did you come to the CAM oncology clinic?

(continued).

Once a month

Twice a month

Thrice a month

Once half a year
$85(22.8)$

7 (1.9)

$4(1.1)$

$7(1.9)$
$123(33.0)$

$17(4.6)$

$4(1.1)$

$8(2.1)$

1. Since the epidemic of COVID-19, have you ever changed your CAM oncologist/therapist?*

No, I have never changed

Basically, but I have once changed

Yes, I have changed

I haven't a specified oncologist/therapist

Other

1. Since the epidemic of COVID-19, how about the cost of the CAM cancer treatment? ${ }^{*}$
$276(74.0)$

$37(9.9)$

$44(11.8)$

$15(4.0)$

$1(0.3)$

$52(13.9)$
Increased

Decreased

No change

I don't know

1. Since the epidemic of COVID-19, have you changed the follow-up pattern?*

Yes

No
15 (4.0)

151 (40.5)

155 (41.6)

Table 4 (continued). 
1. How did you contact your physician?

\begin{tabular}{lll} 
Hospital & $34(100.0)$ & $32(94.1)$ \\
\hline Wechat & $5(14.7)$ & $24(70.6)$ \\
\hline Telephone/message & $0(0.0)$ & $12(35.3)$ \\
\hline Online medical service & $2(5.9)$ & $9(26.5)$ \\
\hline E-mail & $0(0.0)$ & $0(0.0)$ \\
Other & $0(0.0)$ & $0(0.0)$
\end{tabular}

Abbreviations: CAM: complementary and alternative medicine; COVID-19: coronavirus disease 2019.

* Only available for the time after the epidemic of COVID-19.

\# Only available for those who chose YES in question 6.

\section{Discussion}

This study was the first study to evaluate the changes of cancer patients' perception, attitude and clinical practice in CAM during COVID-19. The study revealed that cancer patients showed more interest in CAM and gradually accepted the new medical pattern since the epidemic of COVID-19.

In this study, the usage of CAM by cancer patients (83.3\%) was similar to previous studies in China $(77.6 \%-83.0 \%)[4,5]$, but was much higher than usage in other western countries, including the United States $(33.3 \%)$, Sweden $(26 \%)[2,14,15]$. This is not a surprising finding that CAM is intricately intertwined with the history, culture, and politics of China, which is greatly promoted by the Chinese people. As to the type of cancer, the percentage of lung cancer $(75.4 \%)$ was significantly higher than prior studies $(7.9 \%-33.2 \%)$. The difference might be related to the fact that the department of integrative oncology in the centers are so famous for lung cancer treatment in China, and has a good reputation, which attracts more lung cancer patients. We found statistically significant relationships between CAM use and being older, higher educated, lager income, longer duration, lower ECOG-PS score and having national health insurance, part of which were in line with other surveys $[5,14,16]$. However, from the results of the multivariate analyses, CAM use was predicted only by national health insurance. The differences might be due to the mobility restrictions during the COVID-19 [17], which prevented nonlocal cancer patients from visiting the clinic as usual, so the majority of participants were natives with national health insurance. 
The most common therapies used in this study were Chinese herbal medicine before (89.5\%) / after (90.3\%) the epidemic of COVID-19, which was similar to other studies [18]. However, in stark contrast, most common CAM researches in western countries tend to focus on acupuncture, while few cancer patients use acupuncture in China. As to this study, the usage of acupuncture was very low (3.2\%) and even declined during the COVID-19 (2.4\%). Studies have already shown acupuncture to be a useful adjunct in relieving cancer and treatment-related symptoms $[19,20]$, so oncologists should be encouraged to recommend it to their patients. For the expectations of CAM use, a prior study showed $85.0 \%$ of the cancer patients reported using CAM as an "immune booster" [21]. In this study, the majority of participants reported the same purpose before (69.7\%) / after (71.8\%) the epidemic of COVID-19, as immune system has a close interaction with COVID-19 infection and cancer progression [22, 23]. CAM was quite famous for its regulating immune system effect in cancer patients and fighting against COVID$19[24,25]$, and cancer patients seemed to show more interest $(70.2 \%)$ and confidence $(79.4 \%)$ in CAM treatment during the COVID-19 though their frequency of CAM clinic visit dropped significantly.

With the ongoing COVID-19, the governments and hospitals across the world scrambled to find new medical pattern for outpatient and inpatient visits of cancer patients, ensuring both curative effect and safety. Online medical service pattern was then rising to the forefront [26], and the application in CAM might be a new attempt. A prior study enhanced on telehealth integrative oncology consultations reported telehealth could lower patients' symptom management concerns and provided more herb information, which seemed to be widely accepted [27]. However, in the current study, more than three quarters of the cancer patients did not accept online medical service. Most of them expressed concerns about the accuracy of online diagnosis and treatment with no pulse-taking and discolored image of tongue. As facing the fact that the end to COVID-19 is still not in sight, we find a trend that more patients attempted to accept the advent of online era.

This study has some limitations to be acknowledged. First, the results may not represent all the cancer patients in China, for it was only conducted at five centers in a large metropolis. Besides, more lung cancer patients were observed in the study. Second, COVID-19 prevented many cancer patients from visiting the clinic as usual, especially nonlocal patients and advanced cancer patients, which led to selective bias. Thirdly, 62 invalid questionnaires were excluded for the incompleteness or incorrect filling, which might affect the sample size. Moreover, the survey was mainly focused on the perceptions and attitudes of China's cancer patients on CAM, which of the oncologists still remain unclear.

\section{Conclusion}

China's cancer patients show more interest and confidence in treating cancer by CAM, and the new medical pattern such as medical appointment, has been gradually accepted during the COVID-19 pandemic. However, the cancer patients' perception in CAM and online medical service pattern still need to be improved.

\section{Abbreviations}


CAM: Complementary and alternative medicine; COVID-19: Coronavirus disease 2019; TCM: Traditional Chinese medicine; $\mathrm{NCClH}$ : National Center for Complementary and Integrative Health; ECOG-PS: Eastern Cooperation Oncology Group - performance status; SD: Standard deviation; OR: Odds ratio.

\section{Declarations}

\section{Acknowledgements}

We would like to thank all participants in the study and all the investigators for data collection.

\section{Authors' contributions}

LX, HQZ, YCW and GLY conceived of the survey; ZG and CYW collected the data and conducted the data analysis. YCW and ZG wrote the paper; LX, HQZ and GLY reviewed the manuscript. All authors had read and approved the final manuscript.

\section{Funding}

Not applicable.

\section{Availability of data and materials}

The data used and/or analyzed during the study are available from the corresponding author on reasonable request.

\section{Ethics approval and consent to participate}

The study was approved by Ethics Committee of Yueyang Hospital, Shanghai University of Traditional Chinese Medicine (2020-073).

\section{Consent for publication}

All the authors listed have approved the manuscript and agreed with the submission.

\section{Competing interests}

The authors declare that they have no competing interests.

\section{Author details}

${ }^{1}$ Department of Oncology, Yueyang Hospital of Integrated Traditional Chinese and Western Medicine, Shanghai University of Traditional Chinese Medicine, Shanghai, China. ${ }^{2}$ Department of Medicine, Shanghai Clinical Research Center, Shanghai, China. ${ }^{3}$ Department of Traditional Chinese and Western Medicine, Shanghai Pulmonary Hospital, Tongji University, Shanghai, China. ${ }^{4}$ Department of Traditional Chinese Medicine, Changhai Hospital, Naval Medical University, Shanghai, China. 


\section{References}

1. Sung H, Ferlay J, Siegel RL, Laversanne M, Soerjomataram I, Jemal A, et al. Global Cancer Statistics 2020: GLOBOCAN Estimates of Incidence and Mortality Worldwide for 36 Cancers in 185 Countries. CA Cancer J Clin. 2021;71(3):209-49.

2. Sanford NN, Sher DJ, Ahn C, Aizer AA, Mahal BA. Prevalence and Nondisclosure of Complementary and Alternative Medicine Use in Patients With Cancer and Cancer Survivors in the United States. JAMA Oncol. 2019;5(5):735-7.

3. Yang G, Lee R, Zhang H, Gu W, Yang P, Ling C. National survey of China's oncologists' knowledge, attitudes, and clinical practice patterns on complementary and alternative medicine. Oncotarget. 2017;8(8):13440-9.

4. Yang G, Zhang H, Gan Z, Fan Y, Gu W, Ling C. Discrepant Views of Oncologists and Cancer Patients on Complementary and Alternative Medicine in a Chinese General Hospital. Integr Cancer Ther. 2018;17(2):451-7.

5. McQuade JL, Meng Z, Chen Z, Wei Q, Zhang Y, Bei W, et al. Utilization of and Attitudes towards Traditional Chinese Medicine Therapies in a Chinese Cancer Hospital: A Survey of Patients and Physicians. Evid Based Complement Alternat Med. 2012; doi: 10.1155/2012/504507.

6. Yang G, Zhang H, Yang Y. Challenges and Countermeasures of Integrative Cancer Therapy in the Epidemic of COVID-19. Integr Cancer Ther. 2020; doi: 10.1177/1534735420912811.

7. Xu S, Cheng X, Pan Z, Song Q, Wang Y, Xiong J, et al. Cancer patient management strategy in a Cancer Center of Zhejiang, China during the COVID-19 pandemic. BMC Cancer. 2020;20(1):1194.

8. Chen G, Su W, Yang J, Luo D, Xia P, Jia W, et al. Chinese herbal medicine reduces mortality in patients with severe and critical Coronavirus disease 2019: a retrospective cohort study. Front Med. 2020;14(6):752-9.

9. Shi N, Liu B, Liang N, Ma Y, Ge Y, Yi H, et al. Association between early treatment with Qingfei Paidu decoction and favorable clinical outcomes in patients with COVID-19: A retrospective multicenter cohort study. Pharmacol Res. 2020; doi: 10.1016/j.phrs.2020.105290.

10. Chen J, Lin S, Niu C, Xiao Q. Clinical evaluation of Shufeng Jiedu Capsules combined with umifenovir (Arbidol) in the treatment of common-type COVID-19: a retrospective study. Expert Rev Respir Med. 2021;15(2):257-65.

11. Xiao M, Tian J, Zhou Y, Xu X, Min X, Lv Y, et al. Efficacy of Huoxiang Zhengqi dropping pills and Lianhua Qingwen granules in treatment of COVID-19: A randomized controlled trial. Pharmacol Res. 2020; doi: 10.1016/j.phrs.2020.105126.

12. Yang G, Li M, Zhang H, Yang Y. The Challenge and Management of Clinical Trials in Integrative Cancer during the COVID-19 Pandemic Worldwide. Integr Cancer Ther. 2021; doi: $10.1177 / 1534735421991218$.

13. Zhuang J, Dai X, Wu Q, Cai H, Fu X, Zhang W, et al. A meta-analysis for Lianhua Qingwen on the treatment of Coronavirus disease 2019 (COVID-19). Complement Ther Med. 2021; doi: 
10.1016/j.ctim.2021.102754.

14. Wode K, Henriksson R, Sharp L, Stoltenberg A, Hök Nordberg J. Cancer patients' use of complementary and alternative medicine in Sweden: a cross-sectional study. BMC Complement Altern Med. 2019;19(1):62.

15. Horneber M, Bueschel G, Dennert G, Less D, Ritter E, Zwahlen M. How many cancer patients use complementary and alternative medicine: a systematic review and metaanalysis. Integr Cancer Ther. 2012;11(3):187-203.

16. Nissen N, Lunde A, Pedersen CG, Johannessen $\mathrm{H}$. The use of complementary and alternative medicine after the completion of hospital treatment for colorectal cancer: findings from a questionnaire study in Denmark. BMC Complement Altern Med. 2014;14:388.

17. Jin H, Lu L, Liu J, Cui M. COVID-19 emergencies around the globe: China's experience in controlling COVID-19 and lessons learned. Int J Qual Health Care. 2021; doi: 10.1093/intqhc/mzaa143.

18. Molassiotis A, Fernández-Ortega P, Pud D, Ozden G, Scott JA, Panteli V, et al. Use of complementary and alternative medicine in cancer patients: a European survey. Ann Oncol. 2005;16(4):655-63.

19. Nystrom E, Ridderstrom G, Leffler AS. Manual acupuncture as an adjunctive treatment of nausea in patients with cancer in palliative care-a prospective, observational pilot study. Acupunct Med. 2008;26(1):27-32.

20. Lau CHY, Wu X, Chung VCH, Liu X, Hui EP, Cramer H, et al. Acupuncture and Related Therapies for Symptom Management in Palliative Cancer Care: Systematic Review and Meta-Analysis. Medicine (Baltimore). 2016;95(9):e2901.

21. Boon H, Stewart M, Kennard MA, Gray R, Sawka C, Brown JB, et al. Use of complementary/alternative medicine by breast cancer survivors in Ontario: prevalence and perceptions. $\mathrm{J}$ Clin Oncol. 2000;18:2515-21.

22. Chowdhury MA, Hossain N, Kashem MA, Shahid MA, Alam A. Immune response in COVID-19: A review. J Infect Public Health. 2020;13(11):1619-29.

23. Candeias SM, Gaipl US. The Immune System in Cancer Prevention, Development and Therapy. Anticancer Agents Med Chem. 2016;16(1):101-7.

24. Wang S, Long S, Deng Z, Wu W. Positive Role of Chinese Herbal Medicine in Cancer Immune Regulation. Am J Chin Med. 2020;48(7):1577-92.

25. Wang J, Ge W, Peng X, Yuan L, He S, Fu X. Investigating the active compounds and mechanism of HuaShi XuanFei formula for prevention and treatment of COVID-19 based on network pharmacology and molecular docking analysis. Mol Divers. 2021; doi: 10.1007/s11030-021-10244-0.

26. Bashshur RL, Doarn CR, Frenk JM, Kvedar JC, Shannon GW, Woolliscroft JO. Beyond the COVID Pandemic, Telemedicine, and Health Care. Telemed J E Health. 2020;26(11):1310-3.

27. Narayanan S, Lopez G, Powers-James C, Fellman BM, Chunduru A, Li Y, et al. Integrative Oncology Consultations Delivered via Telehealth in 2020 and In-Person in 2019: Paradigm Shift During the COVID-19 World Pandemic. Integr Cancer Ther. 2021; doi: 10.1177/1534735421999101. 
Strongly agree $\square$ Agree $\square$ Neither agree nor disagree $\square$ Disagree $\square$ strangly disagree

Since the COVID-19, your CAM oncology clinics have been affected

After experiencing the COVID-19, you are willing to learn more about CAM cancer treatment in the future

After experiencing the COVID-19, you agree that CAM is effective in treating cancer

After experiencing the COVID-19, as to the CAM oncology clinics, you agree that the online appointment pattern is better than the previous on-site registration pattern

After experiencing the COVID-19, as to the CAM oncology clinics, you agree that the online medical service pattern will replace the face to face pattern

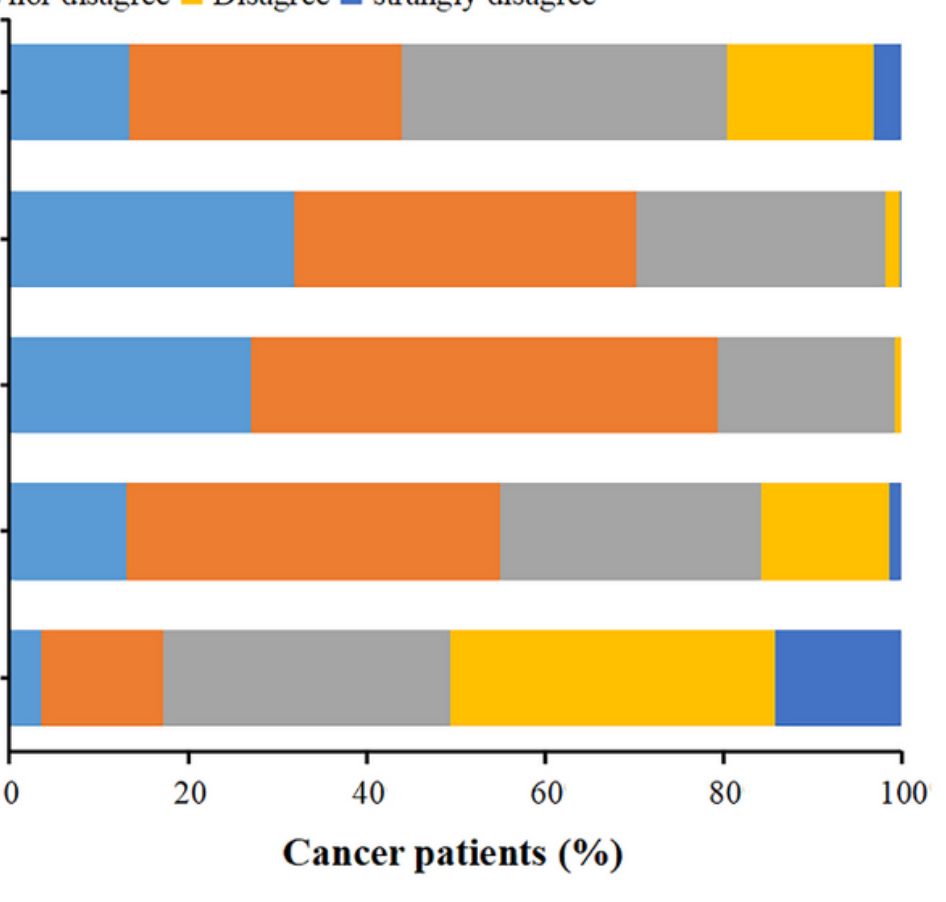

\section{Figure 1}

Attitudes of the cancer patients toward complementary and alternative medicine and medical pattern 